\title{
The Elmore Delay as a Bound for RC Trees with Generalized Input Signals ${ }^{\dagger}$
}

\author{
Rohini Gupta, Byron Krauter*, Bogdan Tutuianu, John Willis and Lawrence T. Pileggi ${ }^{\S}$ \\ The University of Texas at Austin \\ Department of Electrical and Computer Engineering \\ Austin, Texas 78712
}

\begin{abstract}
The Elmore delay is an extremely popular delay metric, particularly for RC tree analysis. The widespread usage of this metric is mainly attributable to it being the most accurate delay measure that is a simple analytical function of the circuit parameters. The only drawbacks to this delay metric are the uncertainty as to whether it is an optimistic or a pessimistic estimate, and the restriction to step response delay estimation.

In this paper, we prove that the Elmore delay is an absolute upper bound on the $50 \%$ delay of an RC tree response. Moreover, we prove that this bound holds for input signals other than steps, and that the actual delay asymptotically approaches the Elmore delay as the input signal rise time increases. A lower bound on the delay is also developed using the Elmore delay and the second moment of the impulse response. The utility of this bound is for understanding the accuracy and the limitations of the Elmore delay metric as we use it for design automation.
\end{abstract}

\section{INTRODUCTION}

$\mathrm{RC}$ trees are commonly used to model digital logic gates and their associated interconnect paths at various stages of the design process. During the early phases of design, simple approximations or delay bounds are often applied since exact solution of an approximate or fluctuating circuit model is superfluous.

The omnipresent Elmore delay [6], or first moment of the impulse response, is the delay approximation of choice for RC trees because of the ease with which it is calculated. In the original work of 1948, Elmore attempted to estimate the 50\% delay of a monotonic step response by the mean of the impulse response. Penfield and Rubinstein [15] proved that RC tree step responses are indeed monotonic, and thereby discovered the popular Elmore delay metric for analyzing gate and interconnect delays. However, because the median of the impulse response is the exact 50\% delay, and Elmore is approximating the median by the mean, Penfield and Rubinstein developed best and worst case bounds on the step response waveform [15].

$\S$ Formerly Lawrence T. Pillage.

* Byron Krauter is with IBM, Austin, TX 78758.

$\dagger$ This work was supported in part by the Semiconductor Research Corporation under contract 94-DJ-343 and the National Science Foundation under contract MIP-9157263.

32nd ACM/IEEE Design Automation Conference ${ }^{\circledR}$

Permission to copy without fee all or part of this material is granted, provided that the copies are not made or distributed for direct commercial advantage, the ACM copyright notice and the title of the publication and its date appear, and notice is given that copying is by permission of the Association for Computing Machinery. To copy otherwise, or to republish, requires a fee and/or specific permission. (C) 1995 ACM 0-89791-756-1/95/0006 \$3.50
These bounds were improved in [18], and later extended to a two time constant approximation in [3]. Some time later higher order moment matching techniques were developed for RLC circuits [16] for which RC trees are an important subset.

But even with higher order approximations with accuracy comparable to SPICE, the Elmore delay remains a popular metric merely for its simplicity. It is used during logic synthesis to estimate wiring delays for approximate tree routes. It is used during performance driven placement and routing because it is the only delay metric which is easily measured in terms of net widths and lengths. The limitations of this model are the uncertainty as to whether it is an optimistic or a pessimistic estimate, and the restriction to it being an estimate only for the step response delay.

In this paper we prove that the Elmore delay value is an absolute upper bound on the $50 \%$ delay of an RC tree. Moreover, we demonstrate that this proof applies not only to the step response, but also to any input forcing function which has a unimodal derivative (e.g. a saturated ramp with finite rise time). With a calculation of the mean and the variance of the impulse response, we also specify an absolute lower bound on the $50 \%$ delay. In addition, we will show that the exact delay approaches the Elmore bound as the variance of the inputsignal derivative increases.

\section{RC TREES AND THEIR APPROXIMATIONS}

\section{A. Interconnect Models}

$\mathrm{RC}$ trees, such as the one shown in Fig.1, have been widely used

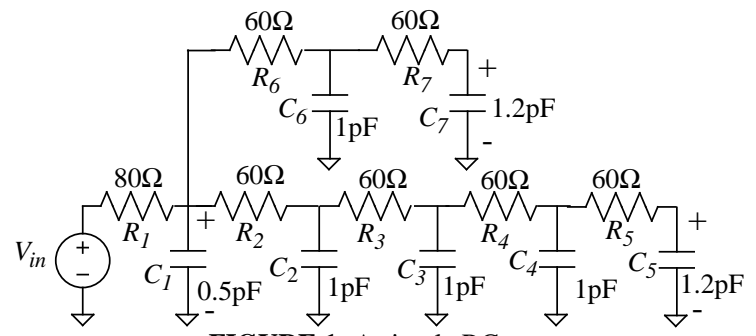

FIGURE 1: A simple RC tree.

for modeling equivalent gate and interconnect circuits. For modeling simplicity, nonlinear drivers are linearized as shown in Fig.1. A great deal of work has been compiled over the last several years regarding these linearized gate models $[1,8,13,19]$. In this paper, however, we will focus on estimating the linearized RC tree delay.

\section{B. The Elmore Delay}

The step and unit impulse responses for the node at capacitor $C_{5}$ of the RC tree in Fig.1 is shown in Fig.2. Since the zero-state step response is the integral of the impulse response $h(t)$, the $50 \%$ point delay of the monotonic step response (nonnegative transfer function) is the time $\tau$ at which $\int_{0}^{\tau} h(t) d t=0.5$. Referring to Fig.2, Elmore pro- 
posed to approximate $\tau$ by the mean of the $h(t)$ distribution.

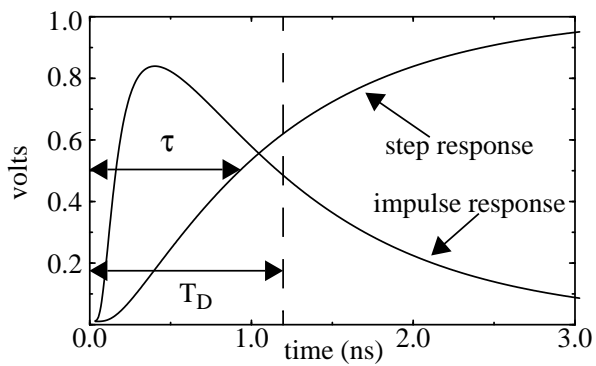

FIGURE 2: The unit step and the unit impulse (scaled by 1e-09) response for the voltage across $\mathrm{C}_{5}$ in Fig.1.

Treating the nonnegative impulse response in Fig.2 as a distribution function, the mean is defined by its first moment, $m_{1}$. Elmore's approximation for the unit step response delay, $T_{D}$, is:

$$
T_{D}=m_{1}=\int_{0}^{\infty} t h(t) d t
$$

where, $\int_{0}^{\infty} h(t) d t=1$. This approximation is valid for a symmetrical function, where the mean is equal to the median, however it is somewhat erroneous for the real impulse response in Fig.2, which is skewed asymmetrically. It is this skew, however, which will allow us to bound the delay $(\tau)$ by the mean $\left(T_{D}\right)$.

\section{Calculating the Elmore Delay}

The Elmore delay is a fitting metric for RC trees because it can be calculated very efficiently for this circuit topology[15,19]. Two $O(N)$ traversals of the tree, where $N$ is the number of nodes in the tree, yield the Elmore delay for node $i$ :

$$
T_{D_{i}}=\sum_{k=1}^{N} R_{k i} C_{k}
$$

where $R_{k i}$ is the resistance of the portion of the (unique) path between the input and node $i$, that is common with the (unique) path between the input and node $k$, and $C_{k}$ is the capacitance at node $k$ [18]. The Elmore delay values at nodes $\mathrm{C}_{1}, \mathrm{C}_{5}$ and $\mathrm{C}_{7}$ for the circuit in Fig.1 are given in column (3) of TABLE 1 .

\begin{tabular}{|c|c|c|c|c|c|c|}
\hline$(1)$ & $(2)$ & $(3)$ & $(4)$ & $(5)$ & $(6)$ & $(7)$ \\
\hline & $\begin{array}{c}\text { Actual } \\
\text { delay } \\
(\mathbf{n s})\end{array}$ & $\begin{array}{c}\text { Elmore } \\
\text { delay, } \boldsymbol{T}_{\boldsymbol{D}} \\
(\mathbf{n s})\end{array}$ & $\begin{array}{c}\text { Lower } \\
\text { bound, } \\
\boldsymbol{T}_{D^{-}}-\sigma(\mathbf{n s})\end{array}$ & $\begin{array}{c}\text { Single pole, } \\
\boldsymbol{T}_{\boldsymbol{D}} \text {.In(2) } \\
(\mathbf{n s})\end{array}$ & $\begin{array}{c}\mathbf{R P H} \\
\text { upper } \\
\text { bound, } \\
\boldsymbol{t}_{\max } \text { (ns) }\end{array}$ & $\begin{array}{c}\mathbf{R P H} \\
\text { lower } \\
\text { bound, } \\
\boldsymbol{t}_{\min }(\mathbf{n s})\end{array}$ \\
\hline $\mathrm{C}_{1}$ & 0.196 & 0.55 & 0 & 0.383 & 0.55 & 0 \\
\hline $\mathrm{C}_{5}$ & 0.919 & 1.2 & 0.2 & 0.83 & 1.32 & 0.51 \\
\hline $\mathrm{C}_{7}$ & 0.45 & 0.75 & 0 & 0.524 & 1.02 & 0.054 \\
\hline
\end{tabular}

TABLE 1: Delay bounds for circuit in Fig.1.

\section{The First Moment of the Impulse Response}

The Elmore delay has also been used as a dominant time constant approximation. Consider the RC tree transfer function

$$
H(s)=\frac{1+a_{1} s+a_{2} s^{2}+\ldots+a_{n} s^{n}}{1+b_{1} s+b_{2} s^{2}+\ldots+b_{m} s^{m}}=m_{0}+m_{1} s+m_{2} s^{2}+\ldots
$$

where $m \geq n$ and $m_{q}$ is the $q$-th coefficient of the impulse response defined as [16]:

$$
m_{q}=\frac{(-1)^{q}}{q !} \int_{0}^{\infty} t^{q} h(t) d t
$$

These coefficients are related to the moments of a distribution function $h(t)$ (from distribution theory) by the $(-1)^{q} / q$ ! term. That is, the $n$-th moment is defined to be $\int_{0}^{\infty} t h(t) d t$. Hereafter, however, we shall refer to $m_{q}$ as the $q$-th moment of $h(t)$.

To understand the connection between the first moment and the dominant pole, the terms $b_{1}$ and $a_{1}$ can be shown [7] to be the sum of the reciprocal poles and zeros respectively:

$$
b_{1}=\sum_{j=1}^{m} \frac{1}{p_{j}} \quad a_{1}=\sum_{j=1}^{n} \frac{1}{z_{j}}
$$

If there are no low frequency zeros, the numerator coefficients, including $a_{1}$, are small and $T_{D} \cong b_{1}$. Now, if one of the time constants (or poles) is assumed to be the dominant one, i.e.

$$
\frac{1}{p_{d}} \gg \frac{1}{p_{j}}, \quad j=1,2, \ldots, m, \quad j \neq d
$$

then $T_{D} \cong 1 / p_{d}$. This dominant time constant approximation is then used to fit a single pole approximation so that:

$$
v(t)=1-e^{-p_{d} t}
$$

Solving (7) for the $50 \%$ point delay effectively scales the Elmore delay approximation by $\ln (2)$, or about 0.7 .

We should point out that this dominant time constant delay prediction can be pessimistic at one node while optimistic at another for the same RC tree. For example, column (5) of TABLE 1 shows the values of $\ln (2) \cdot T_{D}$ at nodes $\mathrm{C}_{1}, \mathrm{C}_{5}$ and $\mathrm{C}_{7}$ for the circuit in Fig.1. Notice that, when compared with the actual delay values in column (1), the response at $C_{5}$ is optimistically predicted by $\ln (2) \cdot T_{D}$ while that at $C_{1}$ is pessimistically predicted. One way to explain this is by the excessive skew in the $h(t)$ distribution for $C_{1}$, which is shown with the step response for this node in Fig.3, as compared with the skew for the response at $C_{5}$ (shown in Fig.2). It can be expected that using $\ln (2) \cdot$ Mean to approximate the median will be vastly different for these two distributions.

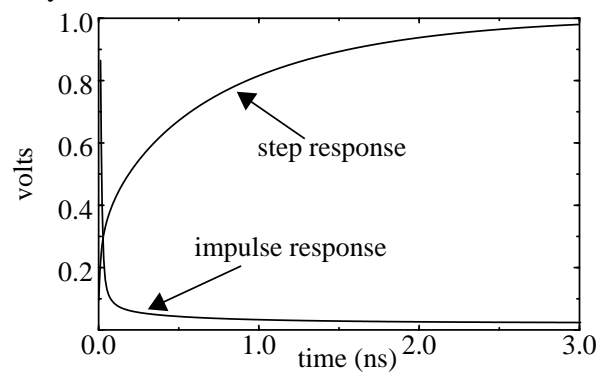

FIGURE 3: The unit step and the unit impulse response (scaled by $4 \mathrm{e}-09$ ) for the voltage across $\mathrm{C}_{1}$ in FIGURE 1.

It is difficult to know when a single pole dominates the low frequency behavior of an RC tree. For this reason, Rubinstein and Penfield established bounds for the RC step response delay.

\section{E. The Rubinstein, Penfield and Horowitz Bounds}

Penfield and Rubinstein were the first to use the Elmore delay to analyze RC trees [15]. Calculating these bounds requires calculating two additional terms in addition to the Elmore delay. All of these terms, however, are obtained with $O(N)$ complexity. The upper and lower bounds, $t_{\max }$ and $t_{\text {min }}$, at the $50 \%$ point for our example in Fig. 1 are given in columns (6) and (7) of TABLE 1. Note that $t_{\text {max }}>T_{D}$ at the loads, $\mathrm{C}_{5}$ and $\mathrm{C}_{7}$, and $t_{\text {max }}=T_{D}$ at the driving 
point, $\mathrm{C}_{1}$. Also note the values of $t_{\min }$ as a lower bound on delay.

In general, one can calculate more moments for the RC tree, and generate a 2-pole [3] or a $q$-pole [16] approximation. Higher order moments are obtained with $O(N)$ complexity too. But for certain applications the Elmore expression is invaluable, and this paper is towards a better understanding of this approximation.

\section{THE ELMORE DELAY AS A BOUND}

Referring back to Fig. 2 and Fig.3, it is apparent that with such an asymmetrical distribution for the impulse response, the mean would not coincide with the median. In this section, we will show that these asymmetric distributions have a "long tail" on the right side of the mode (roughly the maximum value point). Such distributions are said to have positive skew. We will prove that the impulse response for an RC tree is unimodal and positively skewed, then use these two properties to prove that:

\section{Mode $\leq$ Median $\leq$ Mean}

We will further show that (8) holds for any input that has a unimodal derivative and that the mean becomes a better approximation of the median as the rise-time of the input-signal increases. Further in the section, we will also provide a lower bound on the $50 \%$ delay for an RC tree. But first a few definitions:

Definition 1: The mode, $\mathrm{M}$, of a continuous distribution function $f(x)$ is the maximum point of the distribution[4]. A unique mode exists only if $f$ is unimodal and is the solution of

$$
f^{\prime}(x)=\frac{d}{d x} f(x)=0, \quad f^{\prime \prime}(x)=\frac{d^{2}}{d x} f(x)<0
$$

Definition 2: The median, $m$, of a distribution function $f$ is that value of the variate which divides the total frequency into two equal halves[9], i.e.

$$
\int_{-\infty}^{m} f(x) d x=\int_{m}^{\infty} f(x) d x=\frac{1}{2}
$$

Definition 3: The mean, $\mu$, of a distribution function $f$ about the point $x=a$ is defined by

$$
\mu=\int_{-\infty}^{\infty}(x-a) f(x) d x
$$

Definition 4: A density function $h(t)$ is called unimodal, if and only if, there exists at least one value $t=t_{m}$ such that $h(t)$ is nondecreasing for $t<t_{m}$ and nonincreasing for $t>t_{m}$ [17].

Definition 5: Coefficient of skewness for a distribution function is given by $\gamma=\mu_{3} / \sigma^{3}$, where $\sigma=\sqrt{\mu_{2}}$, and $\mu_{2}$ and $\mu_{3}$ are the second and third central moments of the distribution function respectively[4].

LEMMA 1: The impulse response $h(t)$ at any node of an $R C$ tree is a unimodal, positive density function.

Proof: The proof is by induction. For a general RC circuit, it is wellknown that the poles and zeros of the driving point admittance, $Y(s)$, are simple, interlaced and are located on the negative real axis of the $s$-plane[20]. Furthermore, the residues at the poles of $Y(s)$ are real and negative[20]. Therefore, in Fig.4(a), if $v_{i n}(t)$ is an impulse input,

$$
I(s)=Y(s) \quad \text { and }, \quad i(t)=-\sum_{i}\left|k_{i}\right| e^{-p_{i} t}
$$

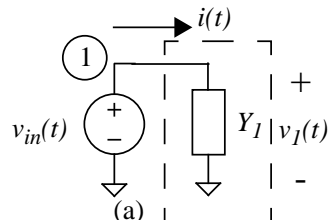

(a) $\llcorner-\lrcorner$

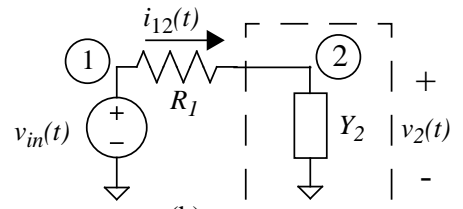

(b)
FIGURE 4: (a) Input node of an RC tree with admittance $\bar{Y}_{l} \overline{\text { at node } 1 .}$

(b)Admittance $Y_{2}$ of an RC tree at the first capacitor node - node 2.

Now, looking at the first downstream node in the $\mathrm{RC}$ tree, in Fig.4(b), clearly, $i_{12}(t)=i(t)$ from Fig.4(a). Therefore,

$$
v_{2}(t)=v_{1}(t)-R_{1} i_{12}(t)
$$

If $v_{1}(t)$ is an impulse, then $v_{2}(t)$ is the impulse response at node 2:

$$
h_{2}(t)=\delta(t)+R_{1} \sum_{i}\left|k_{i}\right| e^{-p_{i} t}
$$

Following Definition 4, it is clear that $h_{2}(t)$ is unimodal.

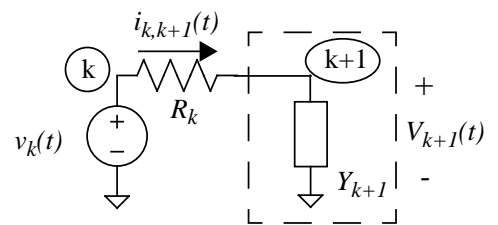

FIGURE 5: Admittance $Y_{k+1}$ of an $\overline{\mathrm{RC}}$ tree at an arbitrary node k+1.

Now consider Fig.5 which shows node $k$ and everything "downstream" of it. To complete our induction argument, we assume that $h_{k}(t)$ is unimodal, and

$$
v_{k+1}(t)=v_{k}(t)-R_{k} i_{k, k+1}(t)
$$

If $v_{k}(t)$ is an impulse, then $h_{k, k+1}(t)$ is the transfer function at node $k+1$ w.r.t. input at node $k$. This has the same form as in (14) and is unimodal. Thus, the transfer function at node $k+1$ w.r.t. node 1 , $h_{k+1}(t)$, is given by:

$$
h_{k+1}(t)=h_{k, k+1}(t) \bullet h_{k}(t)
$$

where $\bullet$ is the convolution operator. Since the convolution of two unimodal density functions is also a unimodal density function [17], we have that $h_{k+1}(t)$ is also unimodal. Thus, $h(t)$ at any node of an $\mathrm{RC}$ tree is a unimodal function. That $h(t)$ is a positive density function has been shown in [18].

\section{LEMMA 2: For the impulse response $h(t)$ at any node of an $R C$} tree, the coefficient of skewness, $\gamma$, is always nonnegative.

Proof: The proof follows an induction-based argument. Following Definition 5, we need to show that for $h(t)$ at any node of an RC tree, $\mu_{3} \geq 0$ and $\mu_{2} \geq 0$. First we show that the coefficient of skewness, $\gamma$, is positive at the first node of an RC tree, and then use the additive property of central moments over convolution to motivate our induction argument.
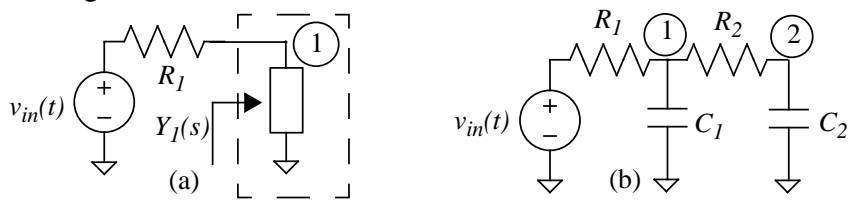

FIGURE 6: (a) Driving point admittance $Y_{l}(s)$ of an RC tree at the first capacitor node. (b)A reduced order $\pi$-model for the $Y_{I}(s)$ in Fig.6(a).

In Fig.6(a), consider a general RC tree for which the first three moments of the driving point admittance, $Y_{1}(s)$ at node 1 , can be used to synthesize a $\pi$-model as shown in Fig.6(b)[12]. Note that this $\pi$-mod- 
el exactly matches the first three moments of the driving point admittance of the original RC circuit. The $\pi$-model parameters are:

$$
\begin{aligned}
& R_{2}=-\frac{\left(m_{3}\left(Y_{1}\right)\right)^{2}}{\left(m_{2}\left(Y_{1}\right)\right)^{3}} \\
& C_{1}=m_{1}\left(Y_{1}\right)-\frac{\left(m_{2}\left(Y_{1}\right)\right)^{2}}{m_{3}\left(Y_{1}\right)} \quad C_{2}=\frac{\left(m_{2}\left(Y_{1}\right)\right)^{2}}{m_{3}\left(Y_{1}\right)}
\end{aligned}
$$

where, $m_{1}\left(Y_{1}\right), m_{2}\left(Y_{1}\right), m_{3}\left(Y_{1}\right)$ are the first three moments of $Y_{1}(s)$.

From distribution theory [4], central moments $\mu_{n}$ of a distribution function are given by:

$$
\mu_{n}=\sum_{k=0}^{n}\left(\begin{array}{l}
n \\
k
\end{array}\right) m_{k}(-\eta)^{n-k}
$$

where, $\eta=m_{1}$ and $m_{k}$ is the $k$-th moment of the distribution function. Thus, for the impulse response of a circuit, we can express the central moments $\mu_{n}$ as a function of the circuit moments $m_{k}$ :

$$
\mu_{2}=2 m_{2}-m_{1}^{2} \text { and } \mu_{3}=-6 m_{3}+6 m_{1} m_{2}-2 m_{1}^{3}
$$

It can be shown that[7] the moments $m_{0}$ through $m_{3}$ of the impulse response $H_{1}(s)$ at node 1 in Fig.6(a) are a function only of the moments $m_{0}$ through $m_{3}$ of the driving point admittance $Y_{1}(s)$ at node 1 . Therefore, the $\pi$-model in Fig.6(b) provides the exact moments $m_{0}$ through $m_{3}$ of the impulse response $H_{l}(s)$.

For the RC circuit in Fig.6(b), we have [16],

$$
\begin{aligned}
& \mu_{2}^{(1)}=2 m_{2}^{(1)}-\left(m_{1}^{(1)}\right)^{2} \\
& =R_{1}^{2}\left(C_{1}^{2}+C_{2}^{2}\right)+2 R_{1}^{2} C_{1} C_{2}+2 R_{1} R_{2} C_{2}^{2} \geq 0 \\
& \mu_{3}^{(1)}=-6 m_{3}^{(1)}+6 m_{1}^{(1)} m_{2}^{(1)}-2\left(m_{1}^{(1)}\right)^{3} \\
& =6 R_{1} R_{2} C_{2}^{2}\left(R_{1}\left(C_{1}+C_{2}\right)+R_{2} C_{2}\right)+2\left(R_{1}\left(C_{1}+C_{2}\right)\right)^{3} \geq 0
\end{aligned}
$$

where, $m_{\mathrm{k}}{ }^{(\mathrm{p})}$ denotes the $k$-th moment at node $p$.

Thus, for the impulse response $h_{1}(t)$ at node 1 in Fig.6(b), from (20) and (21) and Definition 2, we have $\gamma \geq 0$.

Next consider Fig.7 which shows node $k$ and its "downstream" part of the tree. To complete our induction argument, we assume that at node $k$, we have $\mu_{3} \geq 0$ and $\mu_{2} \geq 0$ for $h_{k}(t)$, and hence, $\gamma \geq 0$. If $v_{k}(t)$ is an impulse, then $h_{k, k+1}(t)$ is the transfer function at node $k+1$ w.r.t. the input at node $k$. This has the same form as in Fig.6(a) for which the above argument shows that $\mu_{3} \geq 0$ and $\mu_{2} \geq 0$ (from (20) and (21)). Now, the transfer function at node $k+1$ w.r.t. node 1 , $h_{k+1}(t)$, is given by:

$$
h_{k+1}(t)=h_{k, k+1}(t) \bullet h_{k}(t)
$$

From [4,7], when $m_{0}=1$, we have the property that the second and third central moments add under convolution. Thus,

$$
\begin{aligned}
& \mu_{2}\left(h_{k+1}\right)=\mu_{2}\left(h_{k, k+1}\right)+\mu_{2}\left(h_{k}\right) \geq 0 \\
& \mu_{3}\left(h_{k+1}\right)=\mu_{3}\left(h_{k, k+1}\right)+\mu_{3}\left(h_{k}\right) \geq 0
\end{aligned}
$$

Thus, for $h_{k+1}(t)$, from Definition 5, $\gamma \geq 0$ at all nodes. This proves that for every node in an RC tree, the coefficient of skew-

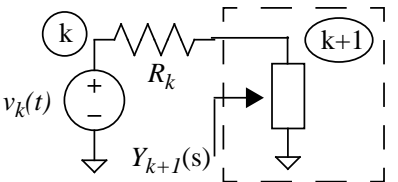

FIGURE 7: Admittance $Y_{\mathrm{k}+1}$ of an RC tree at an arbitrary node $\mathrm{k}+1$. ness, $\gamma \geq 0$.

Now, in the following we use the above two lemmas to prove that the Elmore delay is indeed a bound on the 50\% delay for an RC tree.

THEOREM: For the impulse response $h(t)$ at any node in an $R C$ tree,

$$
\text { Mode } \leq \text { Median } \leq \text { Mean }
$$

Proof: For a unimodal "skewed" distribution function, the mean, median, mode inequality states that these three quantities occur either in alphabetical order or the reverse alphabetical order [9], i.e. either Mean $\leq$ Median $\leq$ Mode or Mode $\leq$ Median $\leq$ Mean. From Lemma 1 and Lemma 2, we have that each node in an RC tree has a unimodal distribution function for which $\gamma \geq 0$. We now prove, by contradiction, that for an RC tree, Mode $\leq$ Median $\leq$ Mean .

For our contradiction argument, let Mean $\leq$ Median $\leq$ Mode hold for any node, $\alpha$, in an RC tree. In a symmetrical distribution, for which the coefficient of skewness, $\gamma$, is exactly zero, the mean, the median and the mode coincide [9,11]. Thus a natural measure of skewness for an asymmetrical distribution is the deviation of the mean from the median, or the mean from the mode. Thus,

$$
S k=\frac{\text { Mean }- \text { Median }}{\sigma}
$$

where, $\sigma=\sqrt{\mu_{2}}$. Thus, at the node $\alpha$, since Mean $\leq$ Median $\leq$ Mode holds, the skewness, $S k \leq 0$. But, from Lemma-2, we have that the coefficient of skewness, $\gamma \geq 0$. Thus, at $\alpha$, either Skew $=0$ or we have a contradiction. In the former case, Mean = Median $=$ Mode, i.e. the distribution is symmetric and the mean and median coincide. And in the latter case, Mode $\leq$ Median $\leq$ Mean .

Since the choice of the node $\alpha$ is arbitrary, the proof is complete. $\square$

We should note at this point that the Elmore Delay, $T_{\mathrm{D}}$, or the mean, $\mu$, of the impulse response approaches the $50 \%$ delay point at nodes further downstream from the source in an RC tree, as discussed further in Section IV.

\section{A. A Lower Bound on Delay}

COROLLARY 1: A lower bound on the 50\% delay for an RC tree is given by

$$
\max (\mu-\sigma, 0)
$$

where $\mu$ is the mean and $\sigma=\sqrt{\mu_{2}}$.

Proof: Consider a positively skewed impulse response $h(t)$, with mean at $t=\mu$. We define another function $H(t)$ as:

$$
H(t)=\int_{-\infty}^{t} h(\zeta) d \zeta
$$

With a simple change in the $\mathrm{x}$-coordinate such that $\tau=t-\mu$, we have $h(\tau)$ such that its mean is at $\tau=0$ in the new coordinate system. Then, using the following inequality from [4](page 256): 


$$
H(\tau) \leq \frac{\sigma^{2}}{\sigma^{2}+\tau^{2}}, \quad \tau<0
$$

For $\tau=-\sigma$, equations (27) and (28) show that

$$
H(-\sigma)=\int_{-\infty}^{\sigma} h(\zeta) d \zeta \leq \frac{\sigma^{2}}{\sigma^{2}+(-\sigma)^{2}}=\frac{1}{2}
$$

Equation (29) states that in the new coordinate system, $\tau=-\sigma$ is less than the median. Thus, in the original coordinate system for $h(t)$ we have that $\mu-\sigma \leq$ Median .

When $\mu \leq \sigma$, since the RC tree system is causal and relaxed[2] and $u(t)=0$ for $t<0$, we have Median $\geq 0$, and hence $\mu-\sigma \leq 0 \leq$ Median. This completes the proof.

Referring back to the example in Fig. 1 and the delay bounds in TABLE 1 , the $\mu-\sigma$ lower bound at $\mathrm{C}_{1}$ equals $t_{\min }$ [18], whereas at $\mathrm{C}_{5}$ and $\mathrm{C}_{7}, t_{\min }$ is a tighter lower bound than $\mu-\sigma$. However, as observed in Section III, the Elmore delay upper bound, $\mu$, becomes a tighter upper bound (as compared to the $50 \%$ bounds in [18]) at the leaf-nodes of an $\mathrm{RC}$ tree as is evident at $\mathrm{C}_{5}$ and $\mathrm{C}_{7}$ in TABLE 1.

\section{B. Approximating the Output Signal Transition Time}

Another measure of practical importance for RC circuits, other than the $50 \%$ delay point, is the rise-time, $T_{\mathrm{R}}$, which may be defined as the 10 to 90 percent transition time[6]. A good measure of $T_{\mathrm{R}}$ is

$$
T_{R}=\sigma=\sqrt{\mu_{2}}
$$

where $\mu_{2}$ is the second central moment of the distribution function $y^{\prime}(t)$. Elmore proposed this value, which he terms the radius of gyration, as a rise-time measure for step-responses [6].

\section{GENERAL INPUT SIGNALS}

It has been shown above that the Elmore delay is an upper bound on the $50 \%$ step response delay. However, when using the Elmore delay to estimate RC interconnect delays, the signal from a digital gate is generally modeled by a saturated ramp. Of course, several models have been developed to characterize the switching gate by a linear resistor and a voltage step for compatibility with the Elmorestep-response model $[1,8,13,19]$ but at the expense of accuracy. One recent work attempts to model high-speed CMOS gates with linear resistors for efficiency, but time varying voltage sources to capture the high-frequency phenomena such as resistance shielding and effective capacitance [5]. Most timing analyzers characterize gate and output signal transition time empirically as a function of load, and then drive the RC tree interconnect model with a voltage that represents this transition time. For these reasons we extend the Elmore bound to consider a non-zero input signal transition time.

\section{A. The Elmore Delay Upper Bound}

COROLLARY 2: For an RC circuit with a monotonically increasing, piecewise-smooth input $u(t)$ such that $u^{\prime}(t)$ is a unimodal symmetric function, Mode $\leq$ Median $\leq$ Mean holds for the output response $y(t)$ at any node.

Proof: The output response $y(t)$ at any node of an $\mathrm{RC}$ tree in response to an input $u(t)$ is given in the Laplace domain by

$$
Y(s)=H(s) \cdot U(s)
$$

where $H(s)$ is the impulse response of the circuit at that node. Using the property of addition under convolution, it can be shown that[7]:

$$
\begin{aligned}
& \mu_{2}\left(y^{\prime}(t)\right)=\mu_{2}(h(t))+\mu_{2}\left(u^{\prime}(t)\right) \\
& \mu_{3}\left(y^{\prime}(t)\right)=\mu_{3}(h(t))+\mu_{3}\left(u^{\prime}(t)\right)
\end{aligned}
$$

From Lemma 2, we know that $\mu_{2}(h(t)) \geq 0$ and $\mu_{3}(h(t)) \geq 0$. From hypothesis, we also have,

$$
\mu_{2}\left(u^{\prime}(t)\right) \geq 0 \quad \text { and } \quad \mu_{3}\left(u^{\prime}(t)\right)=0
$$

From (32) and (33), thus, $\mu_{2}\left(y^{\prime}(t)\right) \geq 0$ and $\mu_{3}\left(y^{\prime}(t)\right) \geq 0$, and from Definition 5, $\gamma\left(y^{\prime}(t)\right) \geq 0$. For $y(t), \therefore$ Median $\leq$ Mean

COROLLARY 3: For a finite sized RC circuit with a monotonically increasing, piecewise-smooth input $u(t)$ with rise-time $t_{p}$ such that $u^{\prime}(t)$ is a unimodal symmetric function, as $t_{r} \rightarrow \infty$, $T_{D} \rightarrow 50 \%$ Delay, i.e. Mean $\rightarrow$ Median .

Proof: From hypothesis, we have that $u^{\prime}(t)$ is a symmetric func-

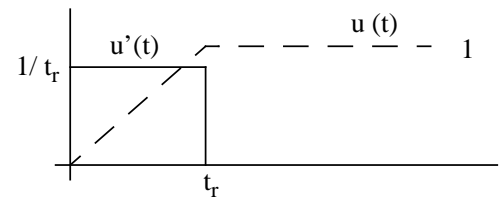

FIGURE 8: Input signal $u(t)$ with rise-time, $t_{r}$ and its derivative, $u^{\prime}(t)$.

tion, $\therefore \mu_{3}\left(u^{\prime}(t)\right)=0$. Also, since $\mu_{2}\left(u^{\prime}(t)\right) \propto t_{r}$,

$$
t_{r} \rightarrow \infty \Rightarrow \mu_{2}\left(u^{\prime}(t)\right) \rightarrow \infty
$$

Since the circuit is finite sized, $\left|\mu_{3}(h(t))\right|<\infty$. Thus, for the output response $y(t)=h(t) \bullet u(t)$ at any node of an RC tree,

$$
\gamma\left(y^{\prime}(t)\right)=\frac{\mu_{3}\left(y^{\prime}(t)\right)}{\left(\mu_{2}\left(y^{\prime}(t)\right)\right)^{3 / 2}} \rightarrow 0 \quad \text { as } t_{r} \rightarrow \infty
$$

Since $\gamma \propto$ Mean - Median , $\gamma \rightarrow 0 \Rightarrow$ Mean $\rightarrow$ Median. Thus, as $t_{r} \rightarrow \infty, T_{D} \rightarrow 50 \%$ Delay.

It is noteworthy here that since $\mu_{3}\left(u^{\prime}(t)\right)=0$, i.e. $u^{\prime}(t)$ is a symmetric function, its mean and median coincide. Further,

$$
\begin{aligned}
\mu\left(y^{\prime}(t)\right)-\mu\left(u^{\prime}(t)\right) & =T_{D} \\
\Rightarrow \int_{0}^{\infty} t y^{\prime}(t) d t-\int_{0}^{\infty} t u^{\prime}(t) d t & =T_{D}
\end{aligned}
$$

where $\mu$ (.) is the mean. Thus, it can be shown that [7]:

$$
\Rightarrow \int_{0}^{\infty}[1-y(t)] d t-\int_{0}^{\infty}[1-u(t)] d t=T_{D}
$$

i.e., the area between the input and the output response equals the Elmore Delay, $T_{\mathrm{D}}[10]$.

\section{B. Delay Curves}

The estimation of the $50 \%$ delay by the Elmore delay as a function of the rise-time of the input signal, as stated in Corollary 3 , is shown in Fig.9 for the RC tree example in Fig.1. As the rise-time of the input signal increases, the delay asymptotically approaches $T_{D}$.

It was observed in Section III that as one moves away from the source, $T_{D}$ (i.e. the mean, $\mu$ ) is a better approximation of the net delay. The proof for Lemma 2 in Section III uses the additive property of the central moments under convolution. Referring to equation (23), for any node $\mathrm{k}, \mu_{2}\left(h_{k}\right), \mu_{3}\left(h_{k}\right) \geq 0$. Furthermore, using equations (20) and (21), it can be shown that $\mu_{2}\left(h_{k, k+1}\right)$ and $\mu_{3}\left(h_{k, k+1}\right)$ form decreasing and hence convergent sequences[7]. 


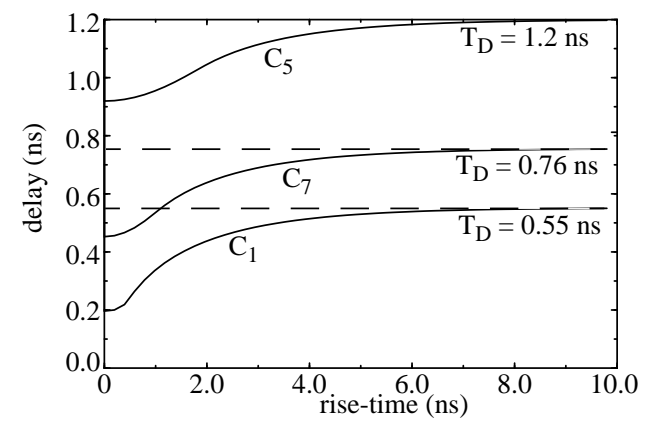

FIGURE 9: Delay curves show that as the rise time of the input signal increases, the delay approaches $T_{D}$.

Thus, as nodes farther away from the source are considered, the values of $\mu_{2}\left(h_{k}\right)$ and $\mu_{3}\left(h_{k}\right)$ start to converge and hence the skew, $\gamma$, converges[7]. The fact that $T_{D}$ is a better approximation of the net delay farther away from the driving point is illustrated here using a 25 node RC tree. For three nodes A, B and C, where A is near the driving point, $\mathrm{B}$ is in the middle of the tree and $\mathrm{C}$ is a leaf-node, the impulse responses are shown in Fig.10. TABLE 2 shows the relative errors (Delay $-T_{D}$ )/Delay for different input signal rise-times. Clearly, from Fig.11, the relative error decreases as a function of the distance from the driving point and input signal rise-time.

\begin{tabular}{|c|c|c|c|c|c|c|c|}
\hline \multirow{2}{*}{ Node } & \multirow{2}{*}{$\begin{array}{c}\text { Elmore } \\
\text { delay }\end{array}$} & \multicolumn{2}{|c|}{ Rise-time $=1 \mathrm{~ns}$} & \multicolumn{2}{c|}{ Rise-time $=5 \mathrm{~ns}$} & \multicolumn{2}{c|}{ Rise-time $=10 \mathrm{~ns}$} \\
\cline { 3 - 8 } & Delay & $\%$ Error & Delay & $\%$ Error & Delay & $\%$ Error \\
\hline $\mathrm{B}$ & $0.02 \mathrm{~ns}$ & $0.01 \mathrm{~ns}$ & $104 \%$ & $0.018 \mathrm{~ns}$ & $11.9 \%$ & $0.019 \mathrm{~ns}$ & $1.54 \%$ \\
\hline $\mathrm{B}$ & $1.13 \mathrm{~ns}$ & $0.72 \mathrm{~ns}$ & $54.7 \%$ & $1.06 \mathrm{~ns}$ & $6.5 \%$ & $1.116 \mathrm{~ns}$ & $0.86 \%$ \\
\hline $\mathrm{C}$ & $1.56 \mathrm{~ns}$ & $1.2 \mathrm{~ns}$ & $29.6 \%$ & $1.48 \mathrm{~ns}$ & $4.8 \%$ & $1.547 \mathrm{~ns}$ & $0.64 \%$ \\
\hline
\end{tabular}

TABLE 2: Delays and relative error at nodes A, B, C along a signal path.

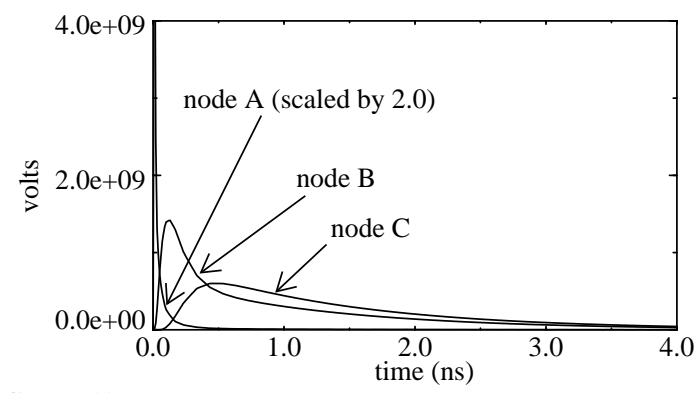

FIGURE 10: Impulse responses at nodes A (driving point), B (middle node) and C (leaf node).

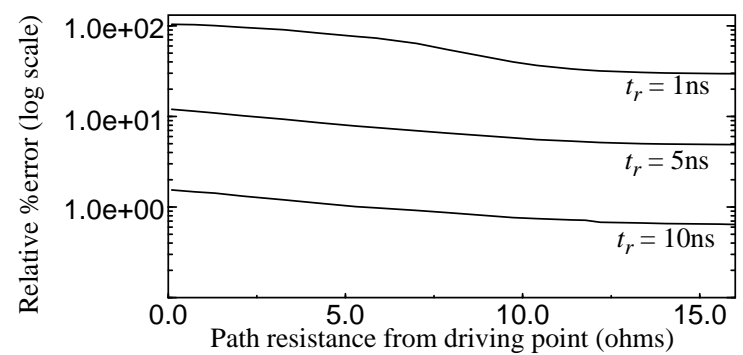

FIGURE 11: Relative error (Delay $-T_{D}$ ) / Delay as function of path resistance from driving point (i.e. distance from driving point).

\section{CONCLUSIONS}

The Elmore delay is an extremely popular delay metric which is used at all levels of electronic circuit design automation. We have proven that this delay measure is an upper bound on the actual $50 \%$ delay of an RC tree response. Moreover, we have proven that this bound holds for input signals other than steps, and that the actual delay asymptotically approaches the Elmore delay as the input signal rise time increases. A lower bound on the delay is also developed as a function of the Elmore delay and the second moment of the impulse response. Improved bounds may be possible with more moments, but moment matching techniques, such as AWE, are preferable when higher order moments are available. The utility of this bound is for understanding the accuracy and the limitations of the Elmore delay metric as we use it as a performance metric.

\section{REFERENCES}

[1] L.M. Brocco. Macromodeling CMOS Circuits for Timing Simulation M.S. thesis, Massachusetts Institute of Technology, June 1987.

[2] C.T. Chen. Linear System Theory and Design. Holt-Saunders Intl. Editions, 1984.

[3] C. Chu and M. Horowitz. Charge-Sharing Models for Switch-Level Simulation. IEEE Trans. on Computer-Aided Design, 6(6):1053-1060, 1987.

[4] Harald Cramer. Mathematical Methods of Statistics. Princeton University Press, 1946.

[5] F. Dartu, N. Menezes, J. Qian and L.T. Pillage. A Gate-Delay Model for High-Speed CMOS Circuits. Proc. of the 31st Design Automation Conference, 1994.

[6] W.C. Elmore. The Transient Analysis of Damped Linear Networks with Particular Regard to Wideband Amplifiers. J. Applied Physics, vol. 19(1), 1948.

[7] R. Gupta, B. Tutuianu and L.T. Pillage. The Elmore Delay as a Bound for RC Trees with Generalized Input Signals. Submitted for publication in the IEEE Transactions on CAD. (Available at WWW site: http:/ /artemis.ece.utexas.edu).

[8] M.A. Horowitz. Timing Models for MOS Circuits. Ph.D. thesis, Stanford University, January 1984.

[9] M.G. Kendall and A. Stuart. The Advanced Theory of Statistics. Vol. I: Distribution Theory. Hafner Publishing Company, 1969.

[10] T.-M. Lin and C.A. Mead. Signal Delay in General RC Networks. IEEE Trans. on Computer Aided Design, CAD-3:331-349, 1984.

[11] H.L. MacGillivray. The Mean, Median, Mode Inequality and Skewness for a class of densities. Australian Journal of Statistics, 23(2), 1981.

[12] P.R. O'Brien and T.L. Savarino. Modeling the driving-point characteristic of resistive interconnect for accurate delay estimation. Proc. IEEE Intl. Conf. Computer-Aided Design, November, 1989.

[13] J.K. Ousterhout. Switch-Level Delay Models for Digital MOS VLSI Proc. of the 21st Design Automation Conference, 1984.

[14] A. Papoulis. Probability, Random Variables and Stochastic Processes. McGraw-Hill International editions, 1984.

[15] P. Penfield and J. Rubinstein. Signal Delay in RC Tree Networks. Proc of the 19th Design Automation Conference, 1981.

[16] L.T. Pillage and R.A. Rohrer. Asymptotic Waveform Evaluation for Timing Analysis. IEEE Transactions on Computer Aided Design, April 1990.

[17] E.N. Protonotarios and O. Wing. Theory of Nonuniform RC Lines. Part II: Analytic Properties in the Time Domain. IEEE Transactions on Circuit Theory, March 1967.

[18] J. Rubinstein, P. Penfield, Jr. and M.A. Horowitz. Signal Delay in RC Tree Networks. IEEE Trans. on Computer Aided Design, CAD-2:202211,1983

[19] C.J. Terman. Simulation Tools for Digital LSI Design. Ph.D. thesis, Massachusetts Institute of Technology, September 1983.

[20] M.E. Van Valkenburg. Introduction to Modern Network Synthesis. John Wiley \& Sons Inc., 1960. 